\title{
A Thermochemistry and Kinetic Study on the Thermal Decomposition of Ethoxyquinoline and Ethoxyisoquinoline
}

\author{
Safinaz H. El-Demerdash ${ }^{1, *}$, Tarek M. El-Gogary, ${ }^{2,3,4}$ and Ahmed M. El-Nahas ${ }^{1, *}$ \\ ${ }^{1}$ Chemistry Department, Faculty of Science, MenoufiaUniversity, 32512 Shebin El-kom, Egypt. \\ ${ }^{2}$ Chemistry Department, Faculty of Science, Jazan University, 2097 Jazan, KSA. \\ ${ }^{3}$ School of Allied Health Sciences, Faculty of Health and Life Sciences, De Montfort University, Leicester, \\ UK. \\ ${ }^{4}$ Chemistry Department, Faculty of Science, Damietta University, 34517 New Damietta, Egypt.
}

\begin{abstract}
Quantum chemical calculations were used to study the production of ethylene from ethoxyquinoline (2-EQ) and ethoxyisoquinoline (1-EisoQ and 3-EisoQ) in gas phase and ethanol at MP2/6$311++\mathrm{G}(2 \mathrm{~d}, 2 \mathrm{p})$. Keto forms of quinoline and isoquinoline (2-EQO, 1-EisoQO, and 3-EisoQO) which are formed through hydrogen migration to nitrogen were studied. Relative energies of decomposition of 2-EQ and 1-EisoQ reveal that the keto form is the dominant form by 1.2 and 3.7 $\mathrm{kcal} / \mathrm{mol}$, respectively. In ethanol, the keto form remains the dominant form for 2-EQ while the hydroxy form of 1-EisoQ is the more stable at the same level of theory. On the other hand, the hydroxy isomer produced from 3-EisoQ is the preferred form in gas phase and ethanol by 8.9 and 4.1 $\mathrm{kcal} / \mathrm{mol}$, respectively. The higher stability of the keto and enol tautomers in ethanol compared to that in gas phase indicates the higher polarity of the formed products. Overall, our calculations show that production of ethylene from 1-EisoQ is more favored than that from 2-EQ and 3-EisoQ.
\end{abstract}

Keywords: Ethoxyquinoline, ethoxyisoquinoline, ethylene formation, potential energy surface, BMK and MP2.

*Corresponding authors. E-mail: amelnahas@hotmail.com (Ahmed El-Nahas), hamdysafinaz@yahoo.com ( Safinaz Hamdy) 


\section{Introduction}

Quinoline or 1-aza-napthalene $\left(\mathrm{C}_{9} \mathrm{H}_{7} \mathrm{~N}\right)$ is one of the most important naturally occurring heterocyclic compounds [1]. Quinoline has attracted considerable attention from scientists for decades due to its pharmaceutical properties such as antimalarial, anti-bacterial, antifungal, anthelmintic, cardiotonic, anticonvulsant, anti-inflammatory and analgesic activity [2-6]. Isoquinoline is the isomer of quinoline in which a $\mathrm{CH}$ group of naphthalene is replaced by a nitrogen atom in positions 2. Their electronic, vibrational, and rotational spectra have been widely investigated due to the relevance of the two compounds to astrophysics, [7] petroleum chemistry [8] as well as medicine [9]. Among the quinoline derivatives, hydroxyquinoline and quinolone have been studied both experimentally and theoretically [10]. Elimination of ethylene from ethoxyquinoline and ethoxyisoquinoline leads to the formation of hydroxyquinoline or quinolone which has a vital importance [11-14].

Over the past two decades, there has been considerable interest in the study of tautomerism of heterocyclic compounds to determine its effect on the chemical and biological properties of molecules. Experimental studies of tautomerism are still a difficult problem in chemistry and molecular biology. Most of the tautomers are not observed in experimental studies due to their low concentration. A detailed analysis of the structure and changes in the geometrical and energetic parameters resulting from hydrogen atom migration would make it possible to understand the different properties of the tautomers.

Hydroxyquinoline is a subunit of many important bioactive molecules so it has a very interesting structure. It is considered as a bifunctional hydrogen-bonding molecule which in aqueous or alcohol solution acts as $\mathrm{H}$-donor at $\mathrm{OH}$ site and an acceptor at the $\mathrm{N}$-atom $[15,16]$. The $\mathrm{OH}$ and aza groups may be also involved in a keto-enol tautomeric equilibrium. Tautomerism plays an important role in organic chemistry, biochemistry, medicinal chemistry, pharmacology, and molecular biology. It is crucial to understand mechanisms of many organic reacions [17]. Some theoretical studies have been done on hydroxyquinoline such as rotation barriers, tautomerism, intramolecular hydrogen bond, solvent effects and proton transfer reactions by density functional theory (DFT) computations [15,18-23]. Filip et.al. studied the molecular structure of 8hydroxiquinoline using B3LYP/6-31+G and PM3 semi-empirical methods [18]. On the other hand, Tokay et. al. studied tautomerism of 2, 3 or 4-hydroxyquinoline derivatives along with their thio 
analogs [19]. Theoretical studies on proton transfer reactions of 8-hydroxyquinoline monomers and dimers have been done [20]. Tautomerism of 4-hydroxyquinoline in gas phase and solution using polarizable continuum method (PCM) has been performed at the B3LYP/6-311++G (d,p) level of theory [21].

Most studies on the tautomerism of 2-substituted quinolin-4-ols and 2-substituted 4quinolones are based largely on the UV, IR and NMR spectroscopic data and to a lesser extent on the semiempirical and ab initio methods [22, 23].

Isoquinoline derivatives are considered biologically important nitrogen hetrocyclic aromatic compounds. They are used as solvent for extraction of several useful medicinally natural products and also as corrosion inhibitor. Quantum mechanical investigation of vibrational and electronic spectra for some 5-substituted isoquinoline has been studied by Suganthi et. al. [24].

Although many chemical and physical phenomena are experimentally studied in condensed phases, the vast majority of quantum chemical calculations for the study of the structure, properties and spectroscopy deal with isolated molecules. There is a lack of theoretical studies on ethoxyquinoline and ethoxyisoquinoline. In the present work, Thermal decomposition of ethoxyquinoline and ethoxyisoquinoline to produce ethylene and different tautomers was studied in gas phase and ethanol solution using polarizable continuum method $(\mathrm{PCM})$ at $\mathrm{BMK} / 6-31+\mathrm{G}(\mathrm{d}, \mathrm{p})$ and MP2/6-311++G(2d,2p) levels of theory. The structural and energetic features of these tautomeric forms and their relative stability were described in this work.

\section{Computational details}

All calculations have been done using the Gaussian-16W program [25]. Geometry optimization of reactants, products and transition states has been performed using Density Functional Theory at the Boese and Martin [26] exchange-correlation functional (BMK) that has been developed for thermochemical kinetics studies [26] in conjunction with the 6- 31+G(d,p) basis set.

The vibrational modes were examined using the ChemCraft application [27] to verify the existence of the transition states. To further ensure that the located transition states connect the desired reactants and products, we performed minimum energy path (MEP) connecting reactants to products through intrinsic reaction coordinate (IRC) calculations in mass-weighted cartesian coordinates with a step size 0.05 and $0.01 \mathrm{amu}^{1 / 2}$ bohr $[28,29]$. With the exception of transition states, vibrational analyses indicated that all of the optimized structures are minima on the potential energy 
surface of the respective systems (all eigenvalues of the force-constant matrices are positive). Solvation effect has been modeled in ethanol using the polarized continuum model (PCM) of Tomasi et. al. [30-33] at the $\mathrm{BMK} / 6-31+\mathrm{G}(\mathrm{d}, \mathrm{p})$ level for the reactant, products and the relevant transition states. Moreover, geometry optimization in solvent for the same systems using PCM has been also performed to examine the effect of solvent on geometries as well as energies. Energy calculations were refined using second-order Møller-Plesset perturbation theory [34], MP2/6-311++G(2d,2p) in gas phase and ethanol.

\section{Results and discussion}

Elimination of ethylene molecule from ethoxyquinoline and ethoxyisoquinoline leads to the formation of the corresponding keto and enol tautomers of quinoline and isoquinoline. The optimized structures of 2-ethoxyquinoline, 2-hydroxy quinoline, 2-quinolone, 1- ethoxyisoquinoline, 1-hydroxy isoquinoline,1-isoquinolone, 3- ethoxyisoquinoline, 3-hydroxy isoquinoline, 3-isoquinolone ( 2-EQ, 2-EQH, 2-EQO, 1-EisoQ, 1-EisoQH, 1-EisoQO, 3-EisoQ, 3-EisoQH, 3-EisoQO), ethylene in gas

phase are given in scheme 1. Some selected geometrical parameters, bond distance of bond breaking and bond making for all transition states and their \% elongation, relative energies and dipole moments for the investigated compounds at the BMK/6-31+G(d) level in the gas phase as well as in ethanol are collected in Tables1-4. The optimized structures of all transition states are shown in figure 1.

The geometries of the investigated compounds were optimized at the BMK/6-31+G(d) level in gas phase and ethanol without any symmetry constraints. Solvent effects were modeled using the polarized continuum model (PCM) of Tomasi et. al. [30-33]. 

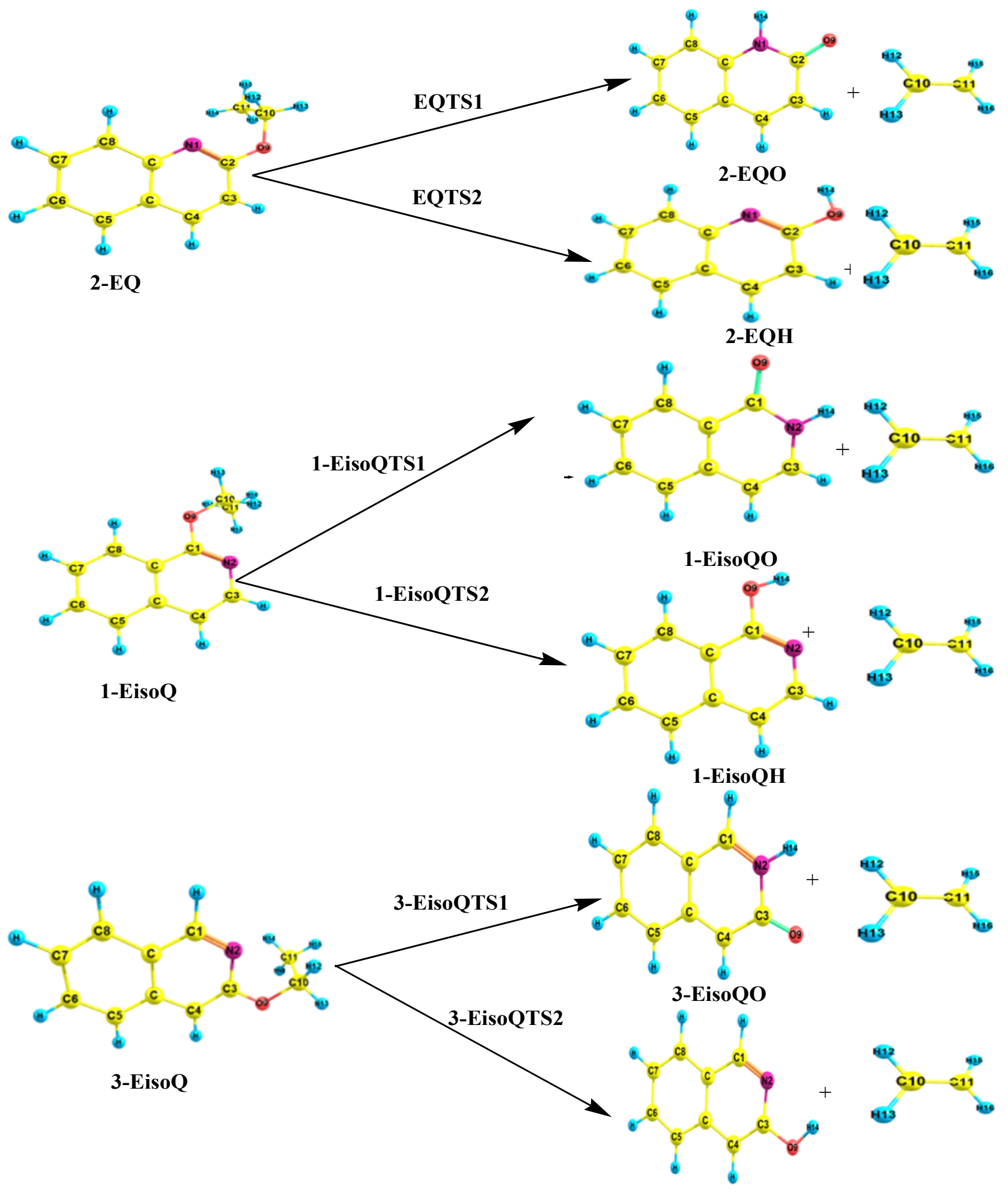

\section{3-EisoQH}

Scheme 1. Unimolecular decomposition of optimized 2-ethoxyquinoline, 1- and 3ethoxyisoquinoline . 


\subsection{Structures}

The keto-enol tautomerism is accompanied by significant changes of the corresponding C-O bond length. The calculated $\mathrm{C}-\mathrm{C}$ bond lengths of the ring are ranging from $1.383 \AA$ to $1.437 \AA$ which lie between the length of the $\mathrm{C}-\mathrm{C}$ single bond $(1.54 \AA)$ and that of the $\mathrm{C}=\mathrm{C}$ double bond $(1.34 \AA)$. Similarly the C-N bond distances in the calculated molecular structures vary from $1.307 \AA$ to $1.369 \AA$ which are also intermediate between those of a $\mathrm{C}-\mathrm{N}$ single bond $(1.48 \AA)$ and the $\mathrm{C}=\mathrm{N}$ double bond $(1.28 \AA)$. Therefore the calculated data suggests an extended $\pi$ electron delocalization over the rings of the system.

All the bond length geometries of the hydroxy form (EQH and EisoQH) are nearly similar to their parent compounds of 2-EQ, 1-EisoQ and 3-EisoQ ( Tables 1 and 2). However, bond angles are different, for example, $\mathrm{N} 1 \mathrm{C} 2 \mathrm{O} 9$ and $\mathrm{O} 9 \mathrm{C} 2 \mathrm{C} 3$ angles are deceased by $2.4^{\circ}$ and $4.2^{\circ}$, respectively. On the other hand, $\mathrm{C} 2 \mathrm{O} 9 \mathrm{H} 14$ angle is increased by $22.7^{\circ}$ in 2-EQ. In addition, for 1-EisoQ angles $\mathrm{C} 1 \mathrm{O} 9 \mathrm{H} 14$ and $\mathrm{N} 2 \mathrm{C} 3 \mathrm{C} 4$ are enlarged by $21.7^{\circ}$ and $5.4^{\circ}$, respectively but $\mathrm{O} 9 \mathrm{C} 1 \mathrm{~N} 2$ angle is decreased by $2.2^{\circ}$. In case of 3-EisoQ, $\mathrm{N} 2 \mathrm{C} 3 \mathrm{O} 9$ and $09 \mathrm{C} 3 \mathrm{~N} 2$ angles are decreased by 2.9 and $2.8^{\circ}$, respectively. Our computed geometrical parameters for isoqunoline are in good agreement with the value calculated at B3LYP/6-311++G(d,p) and HF/6-311++G(d,p) high levels [24].

On the other hand, the geometries of the keto form (2-EQO) reveal that the N1-C2 and C2$\mathrm{C} 3$ bond distances increase by 0.081 and $0.036 \AA$, respectively in 2-EQ (Table 1). The C2-O9 length

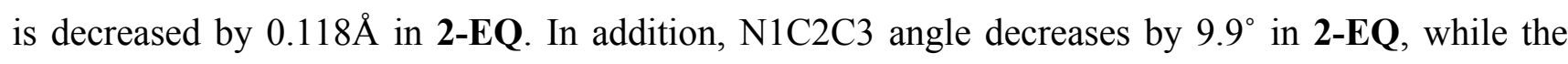
C6C7C8 angle is nearly similar to the parent compound (Table 1). In case of 1-EisoQ, C1-N2 and C3-N2 distances are increased by 0.080 and $0.012 \AA$ but $\mathrm{C} 1-\mathrm{O} 9$ bond length is decreased by $0.116 \AA$. On the other hand, bond lengths (C1-N2 and C3-N2) in 3-EisoQ are enlarged by 0.026 and $0.066 \AA$ but C3-O9 length is shorten by $0.117 \AA$ (Table 2). Detailed distances and angles values of all optimized tautomers at BMK/6-31+G(d,p) are given in Tables 1 and 2. From the results obtained, the geometrical parameters of the keto and hydroxy forms indicate that there are two factors affecting the relative stability of the keto and hydroxy forms; a) the attraction between the $\mathrm{O}-\mathrm{H}$ or $\mathrm{N}-\mathrm{H}$ bonds and the lone pair of the neighboring nitrogen or oxygen atoms, b) the repulsion between the neighboring $-\mathrm{OH}$ and $-\mathrm{NH}$ or $-\mathrm{CH}$ groups as described in former study [35]. In most keto-enol tautomerizations, the equilibrium lies by far toward the keto form indicating that the keto form is usually more stable than the enol form, which can be attributed to the fact that a carbon-oxygen double bond is significantly stronger than a carbon-carbon double bond. 
The keto and enol tautomers are produced from 2-EQ through EQTS1 and EQTS2 transition states as depicted in Figure 1. 1-EisoQ and 3-EisoQ decompose via EisoQTS1 and EisoQTS2 as shown in Figure 1. Frequency analysis shows that the negative frequencies in the transition states correspond to the relevant reaction vector namely $\mathrm{O}-\mathrm{C}, \mathrm{N}-\mathrm{H}$ and $\mathrm{O}-\mathrm{H}$ bonds, respectively. Two possible products, keto and enol tautomers of quinoline and isoquinoline (2-EQO, 2-EQH, 1EisoQO, 1-EisoQH, 3-EisoQO and 3-EisoQH) through two distinguished transition states are formed. Hydrogen migration to either nitrogen atom (keto tautomer, TS1) or oxygen atom (enol tautomer, TS2) results in two reaction channels R1 and R2 respectively. From the optimized geometry of TSs (TS1\&TS2) for reaction channels (R1\&R2), the percentage elongation of C-H (bond breaking) and $\mathrm{O}-\mathrm{H}$ and $\mathrm{N}-\mathrm{H}$ (bond making) with respect to their corresponding equilibrium bond distances are calculated and tabulated in Table 3 . As shown in Table 3 the percentage elongation of bond breaking for R1 in 2-EQ, 1-EisoQ and 3-EisoQ are 28.6, 27.9 and 32.0 which is more than the corresponding percentage elongation of bond making, 25.5, 26.5 and 22.4. This indicates that the corresponding TS1 is closer to the product side and the reaction proceeds via late transition state. In this case, the transition state TS1 more closely resembles the product. The transition state TS1 more closely resembles the reaction side that is higher in energy, in this case the 'product', which means that the reaction is endothermic according to the Hammond Postulate [36]. This means that the keto tautomer ( $\mathrm{R} 1$ reaction channel) is not the kinetically favored product. On the other hand, the percentage elongation of the bond making ( $\mathrm{R} 2$ reaction channel) is much more than the corresponding percentage elongation for bond breaking, 42.0, 46.0, and $42.0 \% v s 20.7,18.2$, and 19.9\%, for 2-EQ, 1-EisoQ, and 3-EisoQ, respectively. This suggests that the respective transition state (TS2) and the barrier of the reaction are near to the corresponding reactants. This means the reaction will proceed via early transition state structure in consonance with Hammond Postulate [36]. According to the Hammond Postulate which states that the transition state in a one-step reaction will, more closely, resemble the side of the reaction that is higher in energy, the transition state TS2 is more related to the reactants and the formed hydroxyl product, enol tautomer, is considered as the kinetically preferred product. A total of 10 points were selected for each reaction near the transition state along the minimum energy path (MEP) defined by intrinsic reaction coordinate (IRC) calculations, at the BMK/6-31+G(d,p)level of theory. This study confirms that each transition state connects the reactants with the relevant products (supplementary material) . 


\subsubsection{Effect of solvent and dipole moment}

One of the most important factors determining the tautomer distribution in different media is the environment. Dipole moments increase on moving from gas phase to the solution. The dipole moments computed in gas phase and ethanol at the BMK/6-31+G(d, p) level are listed in Table 4. Solvation effect has been explored in ethanol using the polarized continuum model (PCM) for the keto and hydroxyl forms and the relevant transition states. Geometry optimization in ethanol for the same systems using PCM has been performed to examine the effect of ethanol on geometries as well as energies. Analysis of calculation results showed that, on going from gas phase to ethanol, there was slight change in the bond lengths of all tautomers. In case of keto forms, there is an elongation of the N1-C2 bond length when changing from gas to ethanol, while C2-O9 bond length was decreased upon solvation. while there are no changes occur for the hydroxy forms. Details of the geometrical features of keto and hydroxy forms and transition states in ethanol can be found in Figures. 1-3 ( Supplementary material).

Table 4 collects the relative energies for keto and enol forms and barrier heights $(\mathrm{kcal} / \mathrm{mol})$ at BMK $/ 6-31+G(d, p)$, and MP2/6-311++G(2p,2d) levels of theory in gas phase and ethanol. The results showed that the keto form has the largest dipole moments in the gas phase and in ethanol. The dipole moment of 2-EQO is 4.55 D and 6.03 D in gas phase and ethanol, respectively at BMK/ 6$31+\mathrm{G}(\mathrm{d}, \mathrm{p})$. In case of 1-EisoQO tautomer, these dipole moment values are $3.85 \mathrm{D}$ and $5.18 \mathrm{D}$ in gas phase and ethanol, respectively. For 3- EisoQO tautomer, the dipole moment is 5.58 D and 7.24 D in gas phase and ethanol, respectively. The order of dipole moment is 3-EisoQO $>$ 2-EQO $>$ 1EisoQO $>$ 3-Eiso3QH $>$ 1-EisoQH $>$ 2-EQH in gas phase and ethanol at the BMK/ 6-31+G(d,p).

The stability increases with increasing solvent polarity. The solvent represented by a polarizable continuum is found to show significant effect on the dipole moments of the individual solute tautomers. Our calculations indicated the predominance of the keto form at MP2/6$311++\mathrm{G}(2 \mathrm{p}, 2 \mathrm{~d})$ (gas and ethanol) for 2-EQ. In gas phase, the keto form of 1-EisoQ is more stable but in ethanol the hydroxy tautomer is the predominant form. In case of 3-EisoQ the hydroxy form is the predominant in gas and ethanol. The intramolecular hydrogen bonding plays an important role in the stability of the highly populated 1-EisoQH and 3-EisoQH in ethanol. Qualitatively, the interaction between different solutes (tautomers) and a constant polar solvent (environmental effects) can be correlated with the magnitude of the solute dipole moment [37]. 
From Table 4, the dipole moment values generally increase in ethanol. This indicates the increase of the charge separation in solvent. It is well known that more polar molecule is stabilized more strongly by polar solvent. The keto tautomers of 2-EQO and 1-EisoQO (6.32 and 5.18 Debye in ethanol) are more strongly stabilized (stabilization energy, $\Delta \mathrm{E}=11.68,9.88 \mathrm{kcal} / \mathrm{mol}$ ) when compared with their enol tautomers, 2-EQH and 1-EisoQH, (2.09 and 2.11 Debye in ethanol) at $\mathrm{BMK} / 6-31+\mathrm{G}(\mathrm{d}, \mathrm{p})$. In gas phase, the 1-EisoQO/1-EisoQH keto/enol stabilization energy is reduced from 16.59 to $9.88 \mathrm{kcal} / \mathrm{mol}$. For 2-EQ reaction channel R1, ethanol lowers the energy of the transition state TS1 more than the energies of reactant and product resulting in a little decrease in reaction energy barrier $(1.03 \mathrm{kcal} / \mathrm{mole})$. On the other hand, for R2 solvent lowers the energy of the reactant and product more than the transition state TS2 resulting in an increase of the reaction energy barrier $(4.04 \mathrm{kcal} / \mathrm{mol})$. For 3-EisoQ at BMK/6-31+G(d,p) (R1\&R2), solvent lowers the energy of the reactant and product more than the transition state (TS1\&TS2) resulting in a decrease of the reaction energy barrier (1.77 and $4.25 \mathrm{kcal} / \mathrm{mol}$ for R1 and R2 respectively). Polar solvent ethanol stabilizes the polar state 3-EisoQTS1 (3.98 Debye) compared with the less polar state 3-EisoQTS2 (2.30 Debye). For 1-EisoQ (R1\&R2), solvent lowers the energy of the transition state TS1\&TS2 more than the reactant and product leading to a decrease of the reaction energy barrier $(1.44$ and $0.42 \mathrm{kcal} / \mathrm{mol}$ for $\mathrm{R} 1$ and $\mathrm{R} 2$ respectively).

\subsection{Energies}

Zero-point-corrected relative energies for ethoxyquinoline (EQ) and ethoxyisoquinoline (EisoQ) are collected in Table 4.

As shown in Figure 2, for 2-EQ the keto form is thermodynamically favored in ethanol and gas phase by 3.7 and $1.2 \mathrm{kcal} / \mathrm{mol}$, respectively at MP2/6-311++G(2d,2p). In addition, at BMK/6$31+\mathrm{G}(\mathrm{d}, \mathrm{p})$ the keto form is still thermodynamically favored in the gas phase and ethanol. Moreover, it is kinetically favored in ethanol but less favored in the gas phase at BMK/6-31+G(d,p) and MP2/6$311++\mathrm{G}(2 \mathrm{~d}, 2 \mathrm{p})$ levels of theory.

Since the possibility of direct tautomerization between products is exist, this process should also be modeled as it affects the concentration of both keto and enol forms. The transition states and the corresponding energy barriers for keto-enol inter-conversion between 2-QO and 2-QH, 1-isoQO and 1-isoQH, 3-isoQO and 3-isoQH were calculated at BMK/6-31+G(d,p) in vaccum and ethanol. The transition states and corresponding energy barriers of the direct keto-enol tautomerization are displayed 
in Figures 10S-12S in the Supplementary Material. In general, the energy barriers are, approximately, half of the barriers for the formation of the keto and enol forms from the reactants. This confirms that the direct inter-conversion is faster than the production of keto and enol forms from ethoxyquinolines. These lower barriers indicate the importance of considering of these direct inter-conversions. ( 2 quinolone and 2 hydroxy quinoline ) and the corresponding energy barriers were calculated at BMK/6$31+\mathrm{G}(\mathrm{d}, \mathrm{p})$ in gas and ethanol. The barrier of direct keto-enol for 2 quinolone and 2 hydroxy quinoline conversion are 33.4 and $38.1 \mathrm{kcal} / \mathrm{mol}$, respectively in gas phase. It confirms that keto form is kinetically favored in the gas phase. In ethanol, keto form is still more kinetically favored by 8.06 $\mathrm{kcal} / \mathrm{mol}$. Direct tautomerization between 1-isoquinlone and 1-isohydroxy quinoline and the corresponding energy barriers shows that keto form is kinetically favored in the gas phase and ethanol by 6.70 and $9.92 \mathrm{kcal} / \mathrm{mol}$, respectively at $\mathrm{BMK} / 6-31+\mathrm{G}(\mathrm{d}, \mathrm{p})$.

The results given in Fig.3 indicate that the keto form of 1-EisoQ is thermodynamically favored in the gas phase at BMK/6-31+G(d,p) and MP2/6-311++G(2d,2p) levels by 6.71 and 3.19 $\mathrm{kcal} / \mathrm{mol}$, respectively but in ethanol the hydroxy form is the more recommended product at MP2/6$311++\mathrm{G}(2 \mathrm{~d}, 2 \mathrm{p})$ level. In solution at $\mathrm{BMK} / 6-31+\mathrm{G}(\mathrm{d}, \mathrm{p})$, the keto form is still the more stable form. Moreover, the hydroxy and keto forms is kinetically slightly favored in ethanol than gas phase.

The hydroxy form of 3-EisoQ, as shown in Fig. 4, is thermodynamically preferable in the gas phase and ethanol. In addition, the hydroxy form is more favored kinetically by 13.0 and 14.6 $\mathrm{kcal} / \mathrm{mol}$ in the gas phase at BMK/6-311+G(d,p) and MP2/6-311++G $(2 \mathrm{~d}, 2 \mathrm{p})$ levels, respectively. Also, it is favored kinetically in ethanol by 12.6 and $10.6 \mathrm{kcal} / \mathrm{mol}$ at MP2/6-311++G(2d, 2p) and $\mathrm{BMK} / 6-31+\mathrm{G}(\mathrm{d}, \mathrm{p})$ levels, respectively.

Comparing the direct tautomerization between 3-isoquinlone and 3-isohydroxy quinoline (Figure 12S) with 3-EisoQ tautomerization it reveals that hydroxy form is kinetically more stable than keto form in gas phase and ethanol in both cases at BMK/6-311+G(d,p).

\section{Conclusion}

A Theoretical study on the production of ethylene from ethoxyquinoline and ethoxyisoquinoline isomers has been performed using MP2/6-311++G(2d,2p) and BMK/6-31+G(d,p) levels in gas phase and ethanol. Keto and enol forms are formed during this process. The results obtained can be summarized as follows:

1. The keto form of $\mathbf{2 - E Q}$ is the most stable form in the gas phase and ethanol. 
2. In ethanol, the stability of the hydroxy tautomer are increased for 3-EisoQ and 1-EisoQ. The corresponding hydroxy tautomers are stabilized only by the attractive interaction of the $\mathrm{O}-\mathrm{H}$ bonds with the lone pairs of the neighboring nitrogen atom in ethanol..

3. The dipole moments of all compounds are affected by solvent.

4. With increasing polarity of medium the dipole moments of the tautomers are increased.

5. the direct tautomerzation between keto and enol show also the keto form is more stable in 2 EQ and1-EisoQ while in case of 3-EisoQ the hydroxy form is the dominant in gas and ethanol.

6. The production of ethylene from 1-EisoQ is more favored than that 2-EQ and 3-EisoQ.

\section{Appendix A. Supplementary material}

Supplementary data associated with this article can be found in the online version,

\section{References}

[1] A. H. Abadi, G. H. Hegazy, A. E. Zaher, Med. Chem. 2005, 13, 5759- 5765.

[2] B. N. Acharya, D. Thavaselvam, M. B. Kaushik, Med. Chem. Res. 2008, 17, 487-494.

[3] H. Assefa, S. Kamath, J. K. Buolamwini, J. Comput. Aided Mol. Des. 2003, 17, 475-493.

[4] A. Baba, N. Kawamura, H. Makino, Y. Ohta, S. Taketomi, T. Sohda, J. Med. Chem.1996, 39, 5176-5182.

[5] M. I. F. Bachiller, C. Perez, G. C. G. Munoz, S. Conde, M. G. Lopez, M. Villarroya, A. G. Garcia, M. I. R. Franco, J. Med. Chem. 2010a, 53, 4927-4937.

[6] M. I. F. Bachiller, C. Perez, G. C. G. Munoz, S. Conde, M. G. Lopez, M. Villarroya, A. G. Garcia, M. I. R. Franco, J. Med. Chem. 2010b, 53, 4927-4937.

[7] Z. Kisiel, O. Desyatnyk, L. Pszczolkowski, S.B. Charnley, P. Ehrenfreund, J. Mol. Spectrosc. 2003, $217,115-122$.

[8] W. V. Steele, D. G. Archer, R. D. Chirico, W. B. Collier, I. A. Hossenlopp, A. Nhuyen, N. K. Smith, B. E. Gammon, J. Chem. Thermodyn. 1988, 20, 1233-1264.

[9] M. Khiavi, J. Vongsvivut, I. Perepichka, A. Mechler, B. R.Wood, D. McNaughton, D. S. Bohle, J. Inorg. Biochem. 2011, 105, 1662-1669 .

[10] Q. S. Li, W. H. Fang, Chem. Phys.Lett. 2003, 367, 637-644. 
[11] J. Jampilek, R. Musiol, M. Pesko, K. Kralova, M. Vejsova, J. Carroll, A. Coffey, J. Finster, D. Tabak, H. Niedbala, Molecules. 14, 2009, 1145-1159.

[12] B. Podeszwa, H. Niedbala, J. Polanski, R. Musiol, D. Tabak, J. Finster, K. Serafin, M. Milczarek, J. Wietrzyk, S. Boryczka, W. Mol, J. Jampilek, J. Dohnal, D. S. Kalinowski, D. R. Richardson, Bioorg. Med. Chem. Lett. 2007, 17, 6138-6141.

[13] R. Musiol, D. Tabak, H. Niedbala, B. Podeszwa, J. Jampilek, Kralova, K. Dohnal, J. Finster, A. Mencel, J. Polanski, Bioorg. Med. Chem. 2008, 16, 4490-4499.

[14] P. C. Appelbaum, P. A. Hunter, Int. J. Antimicrob. Agents. 2000, 16, 5-15.

[15] A. E. Shchavlev, A. N. Pankratov, A. V. Shalabay, Internat. J. of Quantum Chem. 2006, $106,876-886$.

[16] M. Amati, S. Belviso, P. L. Cristinziano, C. Minichino, F. Lelj, I. Aiello, M. La Deda, M. Ghedini, J. Phys. Chem. A. 2007, 111, 13403-13414.

[17] March, J. Advanced Organic Chemistry, Reaction, Mechanism and Structure, 4th ed., John Wiley, New York, 1993.

[18] M. E. Filip, V. I. Humelnicu, I.C. Ghirvu, ACTA CHEMICA IASI. 2009, 17, 85-96.

[19] N. Tokay, C. Ogretir, J. Mol. Struct. (Theochem). 2002, 594, 185-197.

[20] J-Y. Zhao, Z-Y. Zhou, Z-M. Su, Y-Z. Xie, G-Y. Sun, X. Wu, Chinese J. Chem. 2006, 24, 724-730.

[21] H. Zabialah, H. Fatemeh, G. Izatullo, O. Ziyodullo, Chem. Sci. Trans. 2013, 2, 1370-1378.

[22] M. Santo, R. Cattana, J. Silber, J. Spectrochimica Acta A. 2001, 57, 1541-1453.

[23] C. Reichardt, 3rd, WILEY-VCH Verlag GmbH \& Co. KGaA, Weinheim, 2003.

[24] S. Suganthi, V. Kannappan, V. Sathyanarayanamoorthi, R. karunathan, Indian J. Pure Appl. Phys. 2016, 54, 15-34

[25] M. J. Frisch, G. W. Trucks, H. B. Schlegel, G. E. Scuseria, M. A. Robb, J. R. Cheeseman, G. Scalmani, V. Barone, G. A. Petersson, H. Nakatsuji, X. Li, M. Caricato, A. V. Marenich, J. Bloino, B. G. Janesko, R. Gomperts, B. Mennucci, H. P. Hratchian, J. V. Ortiz, A. F. Izmaylov, J. L. Sonnenberg, D. Williams-Young, F. Ding, F. Lipparini, F. Egidi, J. Goings, B. Peng, A. Petrone,T. Henderson, D. Ranasinghe, V. G. Zakrzewski, J. Gao, N. Rega, G. Zheng, W. Liang, M. Hada, M. Ehara, K. Toyota, R. Fukuda, J. Hasegawa, M. Ishida, T. Nakajima, Y. Honda, O. Kitao, H. Nakai, T. Vreven, K. Throssell, J. A. Montgomery, Jr., J. E. Peralta, F. Ogliaro, M. J. Bearpark, J. J. Heyd, E. N. Brothers, K. N. Kudin, V. N. Staroverov, T. A. Keith, R. Kobayashi, 
J. Normand, K. Raghavachari, A. P. Rendell, J. C. Burant, S. S. Iyengar, J. Tomasi, M. Cossi, J. M. Millam, M. Klene, C. Adamo, R. Cammi, J. W. Ochterski, R. L. Martin, K. Morokuma, O. Farkas, J. B. Foresman, and D. J. Fox, Gaussian, Inc., Wallingford CT, 2016.

[26] A. D. Boese, J. M. L. Martin, Chem. Phys. 2004, 121, 3405-3416.

[27] G. A. Zhurko, D. A. Zhurko, ChemCraft version 1.8.

[28] C.Gonzales, HB.Schlegel, J. Phys. Chem. 1989, 90, 2154-2161.

[29] C.Gonzales, HB.Schlegel, J. Phys. Chem.1990, 94, 5507-5523.

[30] S. Miertus, E. Scrocco, J. Tomasi, J. Chem. Phys.1981, 55, 117-129.

[31] J. Tomasi, M. Persico, Chem. Rev.1994, 94, 2027-2094.

[32] R. Cammi, J. Tomasi, .J. Comput. Chem. 1995, 16, 1449-1458.

[33] M. Cossi, V. Barone, R. Cammi, J. Tomasi, J. Chem. Phys. Lett. 1996, 25, 327-335.

[34] C. Møller, M. S. Plesset, J. Phys. Rev.1934, 46, 618-622.

[35] E. Kryachko, M. T. Nguyen, T. H. Zeegers, J. Phys. Chem. A. 2001, 105, 1288-1295.

[36] G. S. A. Hammond, J. Am. Chem. Soc. 1955, 77, 334-338.

[37] J. Leszczynski, J. Phys. Chem. 1992, 96,1649-1653. 